\title{
Micropatterning of Silicon Surface by Direct Laser Interference Lithography
}

\author{
M. Lorens ${ }^{a}$, Y. Zabila ${ }^{b}$, M. Krupiński ${ }^{b}$, M. Perzanowski $^{b}, \mathrm{~K}_{\text {. SuchaneK }}^{b, *}$, \\ K. MARsZaŁeK ${ }^{a}$ And M. MARsZAŁEK ${ }^{b}$ \\ ${ }^{a}$ Faculty of Physics and Applied Computer Science, AGH University of Science and Technology, Kraków, Poland \\ ${ }^{b}$ The Henryk Niewodniczański Institute of Nuclear Physics, Polish Academy of Sciences, Kraków, Poland \\ Direct laser interference lithography is a new and low cost technique which can generate the line- or dot-like \\ periodic patterns over large areas. In the present work, we report on direct fabrication of micrometer structures \\ on Si surface. In the experiments the pulsed high power Nd:YAG laser operating at $1064 \mathrm{~nm}$ wavelength was used. \\ Two-beam configuration with an angle of incidence of $40^{\circ}$ was employed and different laser fluences up to $2.11 \mathrm{~J} / \mathrm{cm}^{2}$ \\ were tested. Areas about $1 \mathrm{~cm}$ in diameter have been processed with a single pulse of $10 \mathrm{~ns}$. The laser treated \\ samples were analyzed by atomic force microscopy to investigate the surface topography and to measure the size \\ and depth of the achieved structures. We observed periodic line-like arrays with grating period of the order of $1 \mu \mathrm{m}$.
}

PACS: $68.55 .-\mathrm{a}, 81.16 .-\mathrm{c}, 81.65 . \mathrm{Cf}$

\section{Introduction}

Over the past several decades surface patterning generated substantial interest due to its application among others in magnetic recording technology $[1,2]$. Demands for the continuous increase in the data storage density bring the challenge to overcome the physical limits for currently used magnetic recording media, via the reduction in domain size.

Numerous techniques have been explored and applied to fabricate small magnetic structures and arrays. The most common approaches use electron beam (EBL) or ion beam lithography (IBL) [3]. However, these techniques involve high cost equipment, multiple steps in a clean room environment and specialized masks. In comparison to these methods laser interference lithography offers the opportunity for fast and flexible realization of periodic structures over macroscopic area in a single processing step [4-8]. In laser interference lithography periodic structures are created either in a photoresist layer covering the material surface [8] or, when high energy lasers are used, directly in the material of interest [4-7]. In the latter technique, called direct laser interference lithography (DLIL), the superposition of coherent laser beams, originating from the same source, locally modifies the properties of irradiated materials. The geometry of the obtained structures could be controlled by changing the incident wavelength $(\lambda)$ as well as the angle between the laser beams $(\theta)$. With two laser beams we get line-like

* corresponding author; e-mail: Katarzyna.Suchanek@ifj.edu.pl structures while three or four beams arranged symmetrically results in dot-like patterns. Unlike other surface texturing methods, DLIL allows a rapid large area fabrication using a single laser pulse.

Here, we report on the use of DLIL to form pattern structures on silicon wafers usually used as a substrate for magnetic films. Two beams DLIL configuration was implemented and different laser fluences were tested. In particular, the effect of nanosecond laser irradiation on silicon with two surface crystalline forms: $\mathrm{Si}(111)$ and $\mathrm{Si}(100)$ were discussed. The surface morphology of fabricated structures was characterized with atomic force microscopy (AFM).

\section{Materials and methods}

Single crystalline silicon wafers with $\mathrm{Si}(111)$ and $\mathrm{Si}(100)$ surfaces were used as a substrate material. The thickness of each sample amounts to $1 \mathrm{~mm}$. The samples were cleaned in ultrasonic bath in organic solvent for several minutes prior to the experiment.

In order to produce line-like surface patterning the samples were irradiated using two beam laser interference arrangements schematically shown in Fig. 1. A pulsed $Q$ -switched Nd:YAG laser (Quantel YAG980) operating at $1064 \mathrm{~nm}$ with pulse duration of $10 \mathrm{~ns}$ and $10 \mathrm{~Hz}$ repetition rate was used as a light source. The output laser beam was directed to the optical beamsplitter which splits the input beam into two coherent beams. A set of mirrors steers the beams towards the substrate with the same angle of incidence. In order to ensure the maximum signal at the interference plane, the optical paths of the 

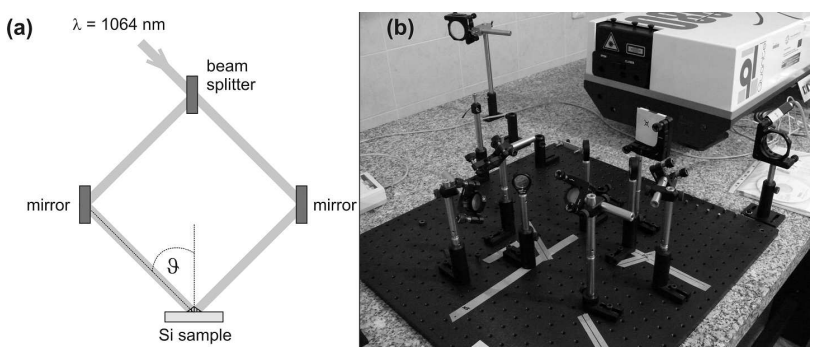

Fig. 1. Two-beam DLIL system: (a) schematic and (b) developed setup.

beams are identical. All structures were produced in a single pulse experiment. The area of irradiation was about $1 \mathrm{~mm}^{2}$. Different laser fluences were used ranging from $1.57 \mathrm{~J} / \mathrm{cm}^{2}$ to $2.11 \mathrm{~J} / \mathrm{cm}^{2}$. A detailed characterization of morphological changes of laser modified areas was done by means of atomic force microscope (AFM) operating in the contact mode.

\section{Results and discussion}

To illustrate the interference pattern generated by two interfering coherent beams, a theoretical simulation using MATLAB 7.5 software was performed before the experiment. Figure 2 shows the calculated spatial distribution of laser intensity corresponding to the interference of two laser beams. High and low intensity values are illustrated in grey and black, respectively on the left in Fig. 2. When the interfering beams with modulated intensity irradiate the sample surface the energy at the interference maxima can cause the spatial heating, melting and even evaporation depending on the energy threshold for a given material. Sinusoidally shaped laser beam modifies the surface by creating the negative image of the interfering beam, e.g. maximum of the intensity corresponds to the topographical minimum. The period $a$ of the interference pattern is given by $a=(\lambda / 2) \sin \theta$, where $2 \theta$ is the angle between the beams. Assuming the $1064 \mathrm{~nm}$ wavelength and an angle of $40^{\circ}$ between the beams we obtained the spacing between the topographical minima of $1136 \mathrm{~nm}$ independently of the laser fluence.

Figure 3 shows AFM images of fabricated periodic structures on $\mathrm{Si}(111)$ wafers. We observed that the topographic structure is well developed on $\mathrm{Si}(111)$ oriented samples. Using relatively high laser fluences (1.57$2.11 \mathrm{~J} / \mathrm{cm}^{2}$ ) we were able to produce well defined line-like array with a grating period of about $1 \mu \mathrm{m}$ which correlates very well with the theoretically predicted value. As can be seen in the AFM cross-section (Fig. 3), the increase of laser fluence did not lead to formation of deeper structures. The apparent height difference between the interference maxima and minima varies between $200 \mathrm{~nm}$ and $20 \mathrm{~nm}$ for all studied samples.

A different behaviour was observed after irradiation of $\mathrm{Si}(100)$ samples (Fig. 4). Within the investigated laser

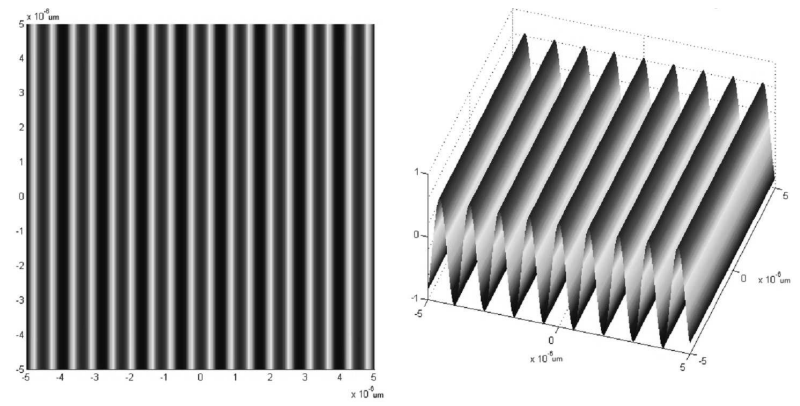

Fig. 2. Spatial distribution of light intensity corresponding to the superposition of two laser beams of the wavelength of $1064 \mathrm{~nm}$ and with the angle between the beams of $40^{\circ}$.

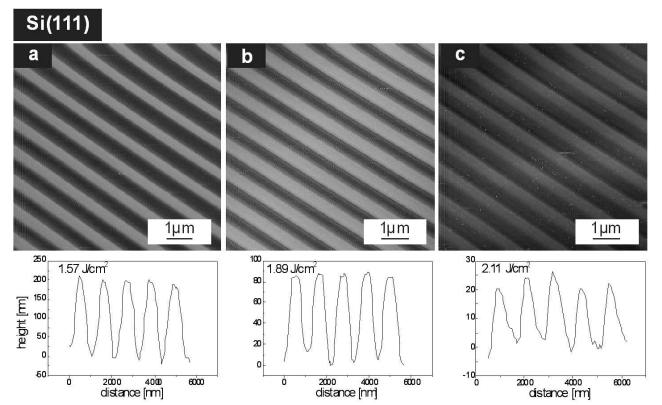

Fig. 3. AFM images of periodic line-like patterns fabricated on $\mathrm{Si}(111)$ substrate with one laser pulse and different laser fluences: (a) $1.57 \mathrm{~J} / \mathrm{cm}^{2}$, (b) $1.89 \mathrm{~J} / \mathrm{cm}^{2}$, (c) $2.11 \mathrm{~J} / \mathrm{cm}^{2}$. Cross-sectional views of examined areas (below).

fluence range, we achieved poor resolution of line-like pattern with rough line edges and irregular shape as compared to $\mathrm{Si}(111)$ wafers.

\section{Conclusions}

In summary, a simple and fast method for fabricating of periodic structures on semiconductor samples was demonstrated. A series of nanosecond pulse experiments

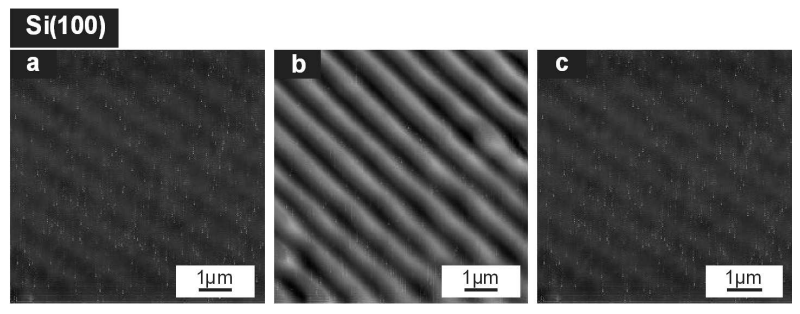

Fig. 4. AFM images of periodic line-like patterns fabricated on $\mathrm{Si}(100)$ substrate with one laser pulse and different laser fluences: (a) $1.57 \mathrm{~J} / \mathrm{cm}^{2}$, (b) $1.89 \mathrm{~J} / \mathrm{cm}^{2}$, (c) $2.11 \mathrm{~J} / \mathrm{cm}^{2}$. Cross-sectional views not shown below the images because readable topographic profile was obtained only for laser fluence of $1.89 \mathrm{~J} / \mathrm{cm}^{2}$. 
have been performed on both $\operatorname{Si}(111)$ and $\operatorname{Si}(100)$ surfaces under ambient conditions. The grating structure with period of the order of $1 \mu \mathrm{m}$ was obtained which is in good agreement with the theoretical calculation.

Moreover, it was observed that the laser induced changes depend on crystal orientation. Our results showed that patterning occurs more readily on $\mathrm{Si}(111)$ than on $\mathrm{Si}(100)$ surfaces. Si(111) samples have well defined structure with uniform periodicity, high contrast, and sharp edges while the interference topography on $\mathrm{Si}(100)$ presents defects or is not well defined. These results suggest that different melting and solidification mechanisms characterise the $\mathrm{Si}(111)$ and $\mathrm{Si}(100)$ surface subjected to nanosecond laser irradiation. Such behaviour was observed earlier in irradiation experiments of crystalline silicon with nanosecond laser pulses $[9,10]$. Once melting, under the action of laser, liquid silicon undergoes the rapid resolidification with very high phase front velocities. These velocities, for the same laser parameters, are different for (111) and (100) oriented surface, which is attributed to the different incorporation of atoms into the lattice. Consequently, the laser fluence for phase transition and the maximum thickness of amorphous layer produced during the process change between $\mathrm{Si}(111)$ and $\mathrm{Si}(100)$ surface. This phenomenon could explain the difference between our results, however more detailed experiments need to be performed, especially because the reports of $[9,10]$ have been devoted to describe the phase transition occurring during nanosecond single beam irradiation without microscoping investigation and patterning of material.

To conclude, the versatility and the facility of controlling with high precision the properties of the produced structures make DLIL technique very attractive for the rapid fabrication of microstructured Si samples. Pre-pattern Si substrates could further serve as a mould for selective magnetic film deposition. In order to obtain structures with smaller features and different periodical architectures an extension of currently reported experimental setup is needed. As in DLIL technique the grating period is dictated by the laser wavelength with additional SHG/THG/FHG laser generator delivering $532 \mathrm{~nm}, 355 \mathrm{~nm}, 266 \mathrm{~nm}$ wavelengths, it is possible to decrease the period dimension and to improve the image resolution.

\section{References}

[1] A. Aktag, S. Michalski, L. Yue, R.D. Kirby, S.-H. Liou, J. Appl. Phys. 99, 093901 (2006).

[2] C. Schuppler, A. Habencht, I.L. Guhr, Appl. Phys. Lett. 88, 012506 (2006).

[3] Y. Cheng, A. Pepin, Electrophoresis 22, 187 (2001).

[4] A. Lasagni, C. Holzapfel, T. Weirich, F. Mucklich, Appl. Surf. Sci. 253, 8070 (2007).

[5] A. Lasagni, F. Mucklich, J. Mater. Proc. Technol. 209, 202 (2009).

[6] Y. Zabila, M. Perzanowski, A. Dobrowolska, M. Kąc, A. Polit, M. Marszałek, Acta Phys. Pol. A 115, 591 (2009).

[7] S. Riedel, M. Schmotz, P. Leiderer, J. Boneberg, Appl. Phys. A 101, 309 (2010).

[8] M. Ellman, A. Rodriguez, N. Perez, M. Echeverrira, Y.K. Verevkin, C.S. Peng, T. Berthou, Z. Wang, S.M. Olaizola, I. Ayerdi, Appl. Surf. Sci. 255, 5537 (2009).

[9] M. Thompson, J. Mayer, A.G. Cullis, H.C. Webber, N.G. Chew, J.M. Poate, D.C. Jacobson, Phys. Rev. Lett. 50, 896 (1983).

[10] J.A. Yater, M.O. Thompson, Phys. Rev. Lett. 63, 2088 (1989). 